Objectives: To asses the extent of structural joint involvement in proximal interphalangeal joints (PIP), hand and wrist: E, BME, S, TS and peritendinitis (PT) by $\mathrm{MRI}$, in patients diagnosed with SLE with hand arthritis or arthralgia.

Methods: All patients with SLE who manifested hand pain and/or swelling in the prior 6 months were consecutively included in the study. They were divided into two groups: arthritis or arthralgia, according to the physical examination by an expert rheumatologist. All patients underwent an MRI with contrast injection on their non-dominant hand. The images obtained were evaluated following RAMRIS criteria extended to PIP and Tenosinovitys score for RA by two expert musculoskeletal radiologists, blind to the groups.

Results: 32 patients were included: arthritis: $n=13$, arthralgia: $n=19$, with a mean age of $50.91 \pm 13.37$ years and a disease evolution time of $10.21 \pm 8.26$ years. The average SLEDAI score $6.30 \pm 3.40$ for the arthritis groups and 3.79 \pm 2.14 for the arthralgia group. The average SLICC score was $0.23 \pm 0.42$ in the arthritis group and $0.1 \pm 0.31$ in the arthralgia group. $E$ was found in 7 patients with arthritis (53.84\%) (15.38\% in PIP, $0 \%$ in hand and $53.84 \%$ in wrist) and in 13 patients with arthralgia (68.42\%) (0\% in PIP, $10.52 \%$ in hand and $68.42 \%$ in wrist). BME was observed in 4 patients with arthritis $(30.76 \%)(7.69 \%$ in PIP, $0 \%$ in hand and $30.76 \%$ in wrist) and in 5 patients with arthralgia (26.31\%) (5.26\% in PIP, 10, 52\% in hand and $15.78 \%$ in wrist). S was observed in 12 patients with arthritis (92.30\%) (61.58 in PIP, $76.92 \%$ in hand and $84.61 \%$ in wrist) and in 8 patients with arthralgia (42.10\%) (31.57\% in PIP, $36.84 \%$ in hand and $36.84 \%$ in wrist). TS was observed in 6 patients with arthritis (46.15\%) (38.46\% in flexor tendons and $23.07 \%$ in extensor tendons) and in 8 patients with arthralgia (42.10\%) (31.57\% in flexor tendons and $21.05 \%$ in extensor tendons). PT was found in 6 patients with arthritis (23.07\%) and in no patient with arthralgia $(0 \%)$

Conclusion: MRI allows us to diagnose musculoskeletal involvement in SLE, morphologically similar to rheumatoid arthritis (erosion, bone marrow edema, synovitis and tenosynovitis), which usually are underestimated on plain radiography. This study also shows the important erosive burden of arthritis in SLE, which has not been well characterised yet. In addition, it demonstrates the underestimation of physical examination to diagnose active inflammatory damage, such as subclinical synovitis, in patients with inflammatory arthralgias.

References:

[1] Mosca M, Tani C, Carli L, Vagnani S, Possemato N, DelleSedie A, et al. The role of imaging in the evaluation of joint involvement in 102 consecutive patients with systemic lupus erythematosus. Autoimmun Rev. 2015; 14: 10-5

[2] Tani C, D’Aniello D, Possemato N, DelleSedie A, Caramella D, Bombardieri S, Mosca M. MRI pattern of arthritis in systemic lupus erythematosus: a comparative study with rheumatoid arthritis and healthy subjects. Skeletal Radiol. 2015;44:261-6

Disclosure of Interests: None declared

DOI: 10.1136/annrheumdis-2020-eular.4825

\section{FRI0163 ANTIPHOSPHOLIPID PATTERNS PREDICT THE RISK OF THROMBOSIS IN SYSTEMIC LUPUS ERYTHEMATOSUS}

S. Demir ${ }^{1,2}$, J. LI$^{1}$, L. Magder ${ }^{3}$, M. A. Petri ${ }^{1} .{ }^{1} J o h n s$ Hopkins University, Department of Rheumatology, Baltimore, United States of America; ${ }^{2}$ Hacettepe University Faculty of Medicine, Department of Pediatric Rheumatology, Ankara, Turkey; ${ }^{3}$ University of Maryland School of Medicine, Department of Epidemiology and Public Health, Baltimore, United States of America

Background: It is well known that Lupus anticoagulant (LAC) positivity is more strongly associated with both arterial and venous thrombosis than either anticardiolipin (aCL) or anti- $\beta 2$ glycoprotein (aB2GPI) antibodies (1). An unanswered question is the contribution of which combinations of positive aPLs to thrombosis risk.

Objectives: We aimed to evaluate which aPL combinations increase the risk of future thrombosis in patients with Systemic Lupus Erythematosus (SLE).

Methods: This prospective analysis included SLE patients who had been tested for all 3 aPLs in Hopkins Lupus Cohort. We constructed a dataset with one record per month of follow up for each patient. Rates of thrombosis for each aPL or each combination of aPL were calculated as the number of thromboses divided by the number of person months at risk and the results are converted to rates per 1000 person-years.

Results: There were 805 patients with a complete profile of 7 aPLs with a total of 73417 person months of follow up. LAC was the most predictive of any $[3.47(1.96,6.14) p<0.0001]$, venous [4.3 $(1.99,9.25) p=0.0002]$, and arterial $[3.37(1.51,7.53) \mathrm{p}=0.0029]$ thrombosis. In individual models, aB2GPI positivity was a significant risk factor for any $[1.90(1.16,3.13)$ $p=0.0113]$ and venous $[2.3(1.23,4.61) p=0.0103]$ thrombosis. When we looked at patients who were LAC positive, and asked if having another positive aPL increase the risk ratio for any/venous/arterial thrombosis, we found that having aB2GPI IgA appeared to add significant risk to any [1.68 (1.01, 2.79) $p=0.044]$, and venous $[2.01(1.02,3.97) p=0.043]$ thrombosis among those with or without LAC (Table 1).

Table 1. Additive effect of other aPLs adjusting for LAC

\begin{tabular}{|c|c|c|c|c|c|c|}
\hline & \multicolumn{2}{|c|}{ ANY thrombosis } & \multicolumn{2}{|c|}{ VENOUS thrombosis } & \multicolumn{2}{|c|}{$\begin{array}{l}\text { ARTERIAL } \\
\text { thrombosis }\end{array}$} \\
\hline & $\begin{array}{l}\text { age adjusted } \\
\text { RR } 95 \%(\mathrm{Cl})\end{array}$ & $\begin{array}{c}\text { P- } \\
\text { value }\end{array}$ & $\begin{array}{l}\text { age adjusted } \\
\text { RR } 95 \%(\mathrm{Cl})\end{array}$ & $p$-value & $\begin{array}{l}\text { age adjusted } \\
\text { RR } 95 \%(\mathrm{Cl})\end{array}$ & p-value \\
\hline \multicolumn{7}{|l|}{$\begin{array}{l}\text { Model 1: LAC + } \\
\text { aCL-G }\end{array}$} \\
\hline LAC (+) vs (-) & $\begin{array}{c}3.78(2.08, \\
6.85)\end{array}$ & $<.0001$ & $\begin{array}{c}5.03(2.25, \\
11.24)\end{array}$ & $<.0001$ & $\begin{array}{c}3.32(1.43 \\
7.73)\end{array}$ & 0.0053 \\
\hline aCL-G (+) vs (-) & $\begin{array}{c}0.64(0.22 \\
1.83)\end{array}$ & 0.4045 & $\begin{array}{c}0.48(0.11 \\
2.14)\end{array}$ & 0.3351 & $\begin{array}{c}1.09(0.31 \\
3.77)\end{array}$ & 0.8962 \\
\hline \multicolumn{7}{|l|}{$\begin{array}{l}\text { Model 2: LAC + } \\
\text { aCL-M }\end{array}$} \\
\hline LAC (+) vs (-) & $\begin{array}{c}3.82(2.13 \\
6.87)\end{array}$ & $<0.0001$ & $\begin{array}{c}4.57(2.07 \\
10.08)\end{array}$ & 0.0002 & $\begin{array}{c}3.69(1.61 \\
8.46)\end{array}$ & 0.0021 \\
\hline aCL-M (+) vs (-) & $\begin{array}{c}0.51(0.16 \\
1.65)\end{array}$ & 0.2576 & $\begin{array}{c}0.68(0.16 \\
2.93)\end{array}$ & 0.6019 & $\begin{array}{c}0.61(0.14 \\
2.64)\end{array}$ & 0.5075 \\
\hline \multicolumn{7}{|l|}{$\begin{array}{l}\text { Model 3: LAC+ } \\
\text { aCL-A }\end{array}$} \\
\hline LAC (+) vs (-) & $\begin{array}{c}3.34(1.88, \\
5.96)\end{array}$ & 0.0001 & $4.14(1.9,9)$ & 0.0003 & $3.29(1.47,7.38)$ & 0.0038 \\
\hline aCL-A (+) vs (-) & $\begin{array}{c}2.33(0.56 \\
9.70)\end{array}$ & 0.2459 & $\begin{array}{c}2.44(0.32 \\
18.4)\end{array}$ & 0.3858 & $\begin{array}{c}2.2(0.29, \\
16.49)\end{array}$ & 0.4415 \\
\hline \multicolumn{7}{|l|}{$\begin{array}{l}\text { Model 4: LAC+ } \\
\text { aB2GPI-G }\end{array}$} \\
\hline LAC (+) vs (-) & $\begin{array}{c}3.52(1.94 \\
6.39)\end{array}$ & 0.0001 & $\begin{array}{l}4.21(1.85 \\
9.57)\end{array}$ & 0.0006 & $3.4(1.48,7.79)$ & 0.0038 \\
\hline $\begin{array}{l}\text { aB2GPI-G (+) } \\
\text { vs }(-) \\
\text { Model 5: LAC+ } \\
\text { aB2GPI-M }\end{array}$ & $\begin{array}{c}0.91(0.32 \\
2.61)\end{array}$ & 0.8655 & $1.1(0.31,3.88)$ & 0.8849 & $\begin{array}{c}0.96(0.22 \\
4.18)\end{array}$ & 0.957 \\
\hline LAC $(+)$ vs $(-)$ & $\begin{array}{c}3.53(1.97, \\
6.31)\end{array}$ & 0.0021 & $4.17(1.9,9.14)$ & 0.0004 & $3.58(1.58,8.1)$ & 0.0023 \\
\hline $\begin{array}{l}\text { aB2GPI-M (+) } \\
\text { vs (-) } \\
\text { Model 6: LAC+ } \\
\text { aB2GPI-A }\end{array}$ & $\begin{array}{c}0.91(0.49 \\
1.73)\end{array}$ & 0.7782 & $\begin{array}{l}1.17(0.51 \\
2.68)\end{array}$ & 0.7101 & $0.74(0.29,1.9)$ & 0.5285 \\
\hline LAC (+) vs (-) & $\begin{array}{c}3.16(1.77, \\
5.65)\end{array}$ & 0.0001 & $\begin{array}{c}3.68(1.68 \\
8.08)\end{array}$ & 0.0012 & $3.19(1.41,7.2)$ & 0.0052 \\
\hline $\begin{array}{l}\text { aB2GPI-A (+) } \\
\text { vs (-) }\end{array}$ & $\begin{array}{c}1.68(1.01 \\
2.79)\end{array}$ & 0.0441 & $\begin{array}{c}2.01(1.02, \\
3.97)\end{array}$ & 0.0434 & $1.4(0.67,2.91)$ & 0.3738 \\
\hline
\end{tabular}

Conclusion: Our study shows that LAC is still the best predictor of risk of any, arterial and venous thrombosis in SLE. Moreover, aB2GPI IgA positivity appeared to add also a significant risk to any and venous thrombosis. Therefore the clinical significance of IgA anti- $\beta 2$ GPI deserves further investigation in SLE patients.

References:

[1] Galli M, Luciani D, Bertolini G, Barbui T. Lupus anticoagulants are stronger risk factors for thrombosis than anticardiolipin antibodies in the antiphospholipid syndrome: a systematic review of the literature. Blood. 2003;101(5) 1827-32.

Acknowledgments: The Hopkins Lupus Cohort was funded by NIH grant number R01 AR069572.

Disclosure of Interests: Selcan Demir: None declared, Jessica Li: None declared, Laurence Magder: None declared, Michelle A Petri Grant/research support from: GSK, Eli Lilly and Company, Consultant of: Eli Lilly and Company

DOI: 10.1136/annrheumdis-2020-eular.1888

\section{FRI0164 BANFF INFLAMMATORY INDICES MAY BE SUPERIOR TO THE NIH SCORING IN PREDICTING CKD PROGRESSION IN LUPUS NEPHRITIS}

M. D. Duong ${ }^{1}$, D. Schwartz ${ }^{2}$, S. Wang ${ }^{3}$, A. R. Broder ${ }^{3}$, B. Goilav ${ }^{1} .{ }^{1}$ Children's Hospital at Montefiore, Nephrology, Bronx, United States of America; ${ }^{2}$ Montefiore Medical Center, Pathology, Bronx, United States of America; ${ }^{3}$ Montefiore Medical Center, Rheumatology, Bronx, United States of America

Background: Chronic kidney disease/end stage renal disease (CKD/ ESRD) from lupus nephritis (LN) is a major cause of morbidity and mortality. 
Advanced tubulointerstitial disease (TID) in LN is a better predicor of renal outcome than glomerular lesions. The current $\mathrm{NIH}$ classification is heavily weighted towards glomerular lesions and only provides a semiqualitative assessment of TID. In contrast, Banff classification of renal allograft pathology provides 6 reproducible scores for TID (inflammation, fibrosis, atrophy). Banff scoring may better predict CKD/ESRD in LN than NIH scores

Objectives: We compared Banff grading vs. $\mathrm{NIH}$ scoring as predictors of CKD progression at 5 years, defined as a decline in estimated glomerular filtration rate (eGFR) $\geq 30 \%$, a strong risk factor for ESRD and mortality

Methods: We included patients with LN class III, IV, V on the index biopsy Jan 2005 and Dec 2018. H\&E/PAS stained slides were reviewed and scored by an experienced pathologist. Six TID Banff scores (0/1 vs. 2/3), NIH activity/chronicity $(\mathrm{Al} / \mathrm{Cl})$ and $\mathrm{NIH}$ interstitial fibrosis/tubular atrophy (IF/TA), tubulointerstitial inflammation (TII) scores (none/mild vs. moderate/severe) were evaluated as predictors of CKD progression using survival analyses

Results: Of the 125 patients, 46 had CKD progression and 20 subsequently developed ESRD. There were no differences between progressors and non-progressors in terms of baseline demographic, clinical data, LN class (Tab 1). Banff ti score (total inflammation) was associated with CKD progression in bivariate and time-dependent analyses. However, NIH TII score and corresponding Banff $\mathrm{i}$ score were not predictive (Tab 2, Fig 1). Overall $\mathrm{NIH} \mathrm{Al} \mathrm{and} \mathrm{Cl}$ were not predictive of CKD progression. Moderate/severe NIH IF/TA was associated with CKD progression as was Banff ci (interstitial fibrosis) score (Tab 2, Fig 2). Banff score for atrophy was not predictive. In a subset of 92 patients with baseline eGFR $\geq 60 \mathrm{ml} /$ $\mathrm{min} / 1.73 \mathrm{~m}^{2}$ only Banff ti score (but not i score or NIH TII, IF/TA) was predictive of CKD progression (Fig 1)

Table 1. Baseline data in patients with/without CKD progression

\begin{tabular}{|c|c|c|c|}
\hline & $\begin{array}{c}\text { Progressors }^{1} \\
n=46\end{array}$ & $\begin{array}{c}\text { Non-progressors } \\
n=79\end{array}$ & $\mathrm{p}$ \\
\hline \multicolumn{4}{|l|}{$\mathrm{n}(\%)$ or median (IQR) } \\
\hline Female & $36(78)$ & $69(87)$ & 0.18 \\
\hline Age (years) & $26(20-41)$ & $29(21-43)$ & 0.53 \\
\hline$<18$ years & $7(15)$ & $13(16)$ & 0.86 \\
\hline Race & & & 0.82 \\
\hline White & $4(9)$ & $4(5)$ & \\
\hline Black & $18(39)$ & $35(44)$ & \\
\hline Asian & $1(2)$ & $1(1)$ & \\
\hline Unknown & $23(50)$ & $39(50)$ & \\
\hline Diabetes & $5(11)$ & $3(4)$ & 0.12 \\
\hline Hypertension & $33(72)$ & $55(70)$ & 0.8 \\
\hline eGFR ml/min/1.73m² & $96.1(56.6-117.8)$ & $88.9(47-117)$ & 0.51 \\
\hline $\mathrm{C} 3 \mathrm{mg} / \mathrm{dL}$ & $71(52-88)$ & $67.5(43-94)$ & 0.58 \\
\hline $\mathrm{C} 4 \mathrm{mg} / \mathrm{dL}$ & $12.65(9-23)$ & $12(7-20)$ & 0.48 \\
\hline \multirow[t]{2}{*}{ Anti-ds DNA titer IU } & 108.7 & 151.3 & 0.57 \\
\hline & $(36.6-194.9)$ & $(39.2-200)$ & \\
\hline Total SLEDAI & $11(8-16)$ & $12(8-16)$ & 0.47 \\
\hline Renal SLEDAI & $8(4-12)$ & $8(4-12)$ & 0.9 \\
\hline LN & & & \\
\hline Proliferative $\mathrm{GN}^{2}$ : III, IV & $18(39)$ & $30(38)$ & 0.15 \\
\hline Nonproliferative GN: V & $9(20)$ & $27(34)$ & \\
\hline Mixed GN: $\mathrm{V}$ and III/IV & $19(41)$ & $22(28)$ & \\
\hline
\end{tabular}

${ }^{1}$ Progressors: LN patients with eGFR decline $\geq 30 \%$ within 5 years. ${ }^{2} \mathrm{GN}$ : glomerulonephritis

Table 2. NIH and Banff scores with/without progressors

\begin{tabular}{lccc}
\hline & $\begin{array}{c}\text { Progressors } \\
\mathrm{n}=46\end{array}$ & $\begin{array}{c}\text { Non- } \\
\text { progressors } \\
\mathrm{n}=79\end{array}$ & $\mathrm{p}$ \\
& & & \\
\hline $\mathrm{n}(\%)$ or median (IQR) & & & \\
$\mathrm{NIH}$ & & $1(0-3)$ & 0.61 \\
Overall AI & $1(0-4)$ & $3(3.8)$ & 0.62 \\
$\mathrm{Al} \geq 11$ & $1(2)$ & $2(0-3)$ & 0.33 \\
Overall CI & $3(0-5)$ & $28(35.4)$ & 0.07 \\
Cl $\geq 3$ & $24(52)$ & $4(5)$ & 0.05 \\
Moderate/severe TII & $7(15.2)$ & $15(19)$ & 0.049 \\
Moderate/severe IF/TA & $16(35)$ & & \\
Banff & & 0 & \\
Tubulitis: t & 0 & $5(6.3)$ & 0.9 \\
Interstitial inflammation: i 2/3 & $3(6.5)$ & $12(15.2)$ & 0.01 \\
Total inflammation: ti 2/3 & $16(34.8)$ & $16(20.3)$ & 0.07 \\
Tubular atrophy: ct 2/3 & $16(34.8)$ & $15(19)$ & 0.03 \\
Interstitial fibrosis: ci 2/3 & $17(37)$ & $\mathrm{n}=51^{*}$ & 0.09 \\
Inflammation in area of interstitial fibrosis and/or tubular & $\mathrm{n}=26^{*}$ & $25(49)$ & \\
atrophy: i-IFTA 2/3 & $18(69)$ & $25(3)$ &
\end{tabular}

* Biopsy number is smaller due to inability to apply score to biopsies without areas of fibrosis/ tubular atrophy

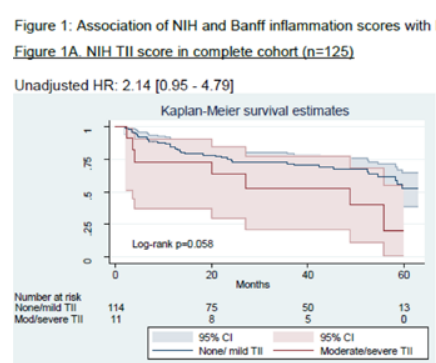

Figure 1C. NIH TII score in sub-cohort with baseline eGFR $260 \mathrm{~m} / \mathrm{min} / 1.73 \mathrm{~m}^{2}(\mathrm{n}=92)$

Unadjusted HR: $1.65[0.39-6.91]$

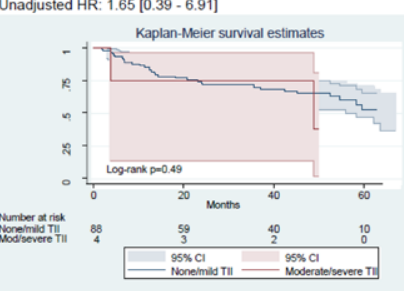

Figure 18. Banfft ti score in complete cohort ( $n=125$ ) Unadjusted HR: $2.96[1.6$ - 5.48]

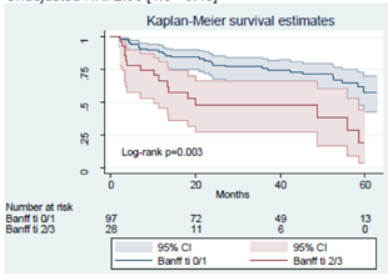

Figure 1D. Banff ti score in sub-cohort with baseline eGFR $260 \mathrm{~m} / \mathrm{min} / 1.73 \mathrm{~m}^{2}(\mathrm{n}=92)$

Unadjusted HR: 3.82 [1.61 - 9.06$]$

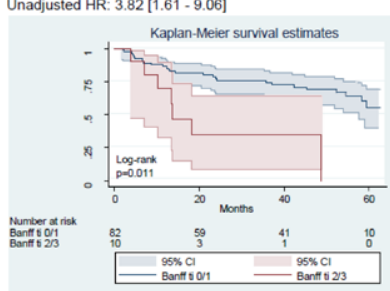

Figure 2: Association of NIH and Banff fibrosis scores with ESRD progression

Figure 2A. NIH IF $T$ A score in complete cohort $(n=125$

Unadjusted HR: 2.12 [1.16 - 3.91] Kaplan-Meler survival estimates

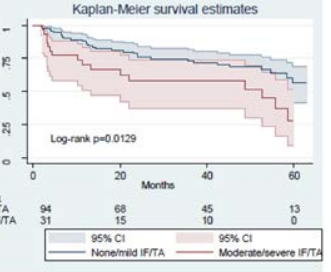

Figure 2B. Banff ci score in complete cohort ( $n=125$ ) Unadjusted HR: 2.27 [1.25 - 4.15]

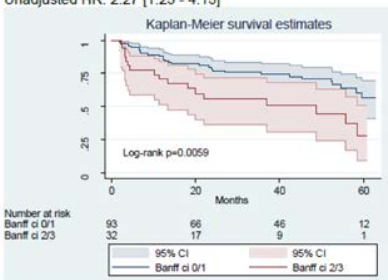

Figure 2C. NIH IFITA score in sub-cohort with eGFR $260 \mathrm{mH}$ $\min / 1.73 \mathrm{~m}^{2}(n=92)$

Unadjusted HR: 1.76 [0.67 - 4.58]
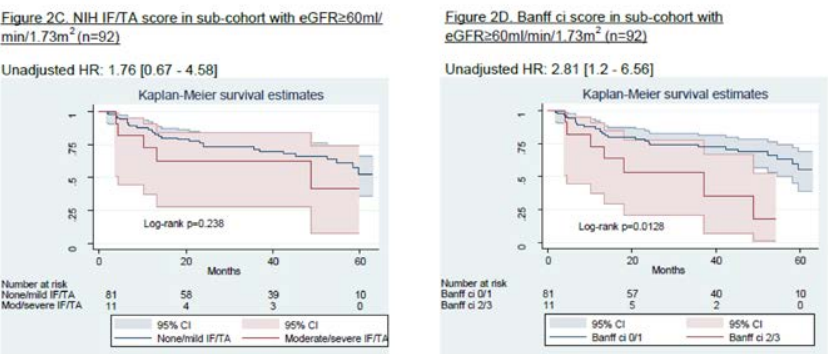

Conclusion: Banff inflammation scores may be superior predictors of CKD/ ESRD progression at 5 years, compared to the currently used NIH classification. Detection of inflammation by Banff scores may allow earlier interventions to prevent ESRD

Disclosure of Interests: None declared

DOI: 10.1136/annrheumdis-2020-eular.1534

\section{FRI0165 RISK OF CKD IN MEMBRANOUS AND PROLIFERATIVE LUPUS NEPHRITIS - ANALYSIS OF A NATIONWIDE MULTICENTRE COHORT}

F. Farinha ${ }^{1}$, S. C. Barreira ${ }^{2}$, M. Couto ${ }^{3}$, M. Cunha ${ }^{4}$, D. Fonseca ${ }^{5}$, R. Freitas ${ }^{4}$,

L. Inês ${ }^{6}$, M. Luis ${ }^{6}$, C. Macieira ${ }^{2}$, A. R. Prata ${ }^{6}$, J. Rodrigues ${ }^{7}$, B. Santos ${ }^{8}$,

R. Pinheiro Torres ${ }^{9}$, R. J. Pepper ${ }^{10}$, A. Rahman ${ }^{1}$, M. J. Santos ${ }^{4} .{ }^{1}$ UCL,

Rheumatology, London, United Kingdom; ${ }^{2} H S M-C H L N$, Rheumatology, Lisbon,

Portugal; ${ }^{3} \mathrm{CHTV}$, Rheumatology, Viseu, Portugal; ${ }^{4} \mathrm{HGO}$, Rheumatology, Almada,

Portugal; ${ }^{5} \mathrm{CHVNG/E}$, Rheumatology, VN Gaia, Portugal; ${ }^{6} \mathrm{CHUC}$, Rheumatology,

Coimbra, Portugal; ${ }^{7}$ ULSAM, Rheumatology, Ponte de Lima, Portugal; ${ }^{8} \mathrm{CHBV}$,

Rheumatology, Aveiro, Portugal; ${ }^{9} \mathrm{HEM}$ - CHLO, Rheumatology, Lisbon, Portugal;

${ }^{10}$ UCL, Nephrology, London, United Kingdom

Background: Lupus nephritis (LN) is one of the most severe manifestations of Systemic Lupus Erythematosus.

Objectives: 1) To compare proliferative (PLN), membranous (MLN) and mixed LN regarding clinical and laboratory presentation. 2) To investigate predictors of progression to chronic kidney disease (CKD). 